nahmen und an der Ausarbeitung allfälliger Korrekturen.

Es eröffnet sich hier also ein weites theoretisches und praktisches Arbeitsfeld für den Kulturgeographen, der sich mit den alpinen, bzw. mit wirtschaftlich benachteiligten Gebieten befaßt.

\section{Anmerkungen}

1 Unterorganisation der CEA (Confédération Européenne de l'Agriculture), Sekretariat in $\mathrm{CH}-5200$ Brugg, Schweiz.
2 Dr. A. Müller: Probleme der Nebenerwerbsbetriebe in den Berggebieten. In: "Bericht zur 11. Arbeitstagung der Europäischen Arbeitsgemeinschaft für wirtschaftliche und soziale Probleme der Berggebiete». Brugg, Juni 1971.

3 Diskussionsbeitrag.

${ }^{4}$ Brugg, 1. Juli 1971.

5 P. Salat: Die Berggebiete im Dienste der Gesellschaft, a. a. O.

${ }^{6}$ Dr. W. Kämpfen, Direktor der Schweizerischen Verkehrszentrale an deren Jahresversammlung (laut Tages-Anzeiger, Zürich, 22. Juli 1971).

\title{
Geologischer Atlas der Schweiz 1:25000
}

Die nachfolgende, in Form einer Liste und Abbildung gegebene Zusammenstellung vermittelt den momentanen Stand des von der Schweizerischen Geologischen Kommission herausgegebenen "Geologischen Atlasses». Der Verkaufspreis beträgt für Blätter auf Papier Fr. 18.65 bzw. für Blätter auf Syntosil (von Blatt Nr. 43 an) Fr. 20.70; das Erläuterungsheft ist im Preis inbegriffen. Die vergriffe- nen Kartenblätter sind mit einem Stern $\left({ }^{*}\right)$ versehen.

Verkauf im Buchhandel oder durch den Kommissionsverlag, Kümmerly \& Frey AG, Bern, bei dem auch der Verkaufskatalog 1971, der die gesamten Publikationen der Geologischen und der Geotechnischen Kommission enthält, gratis bezogen werden kann.

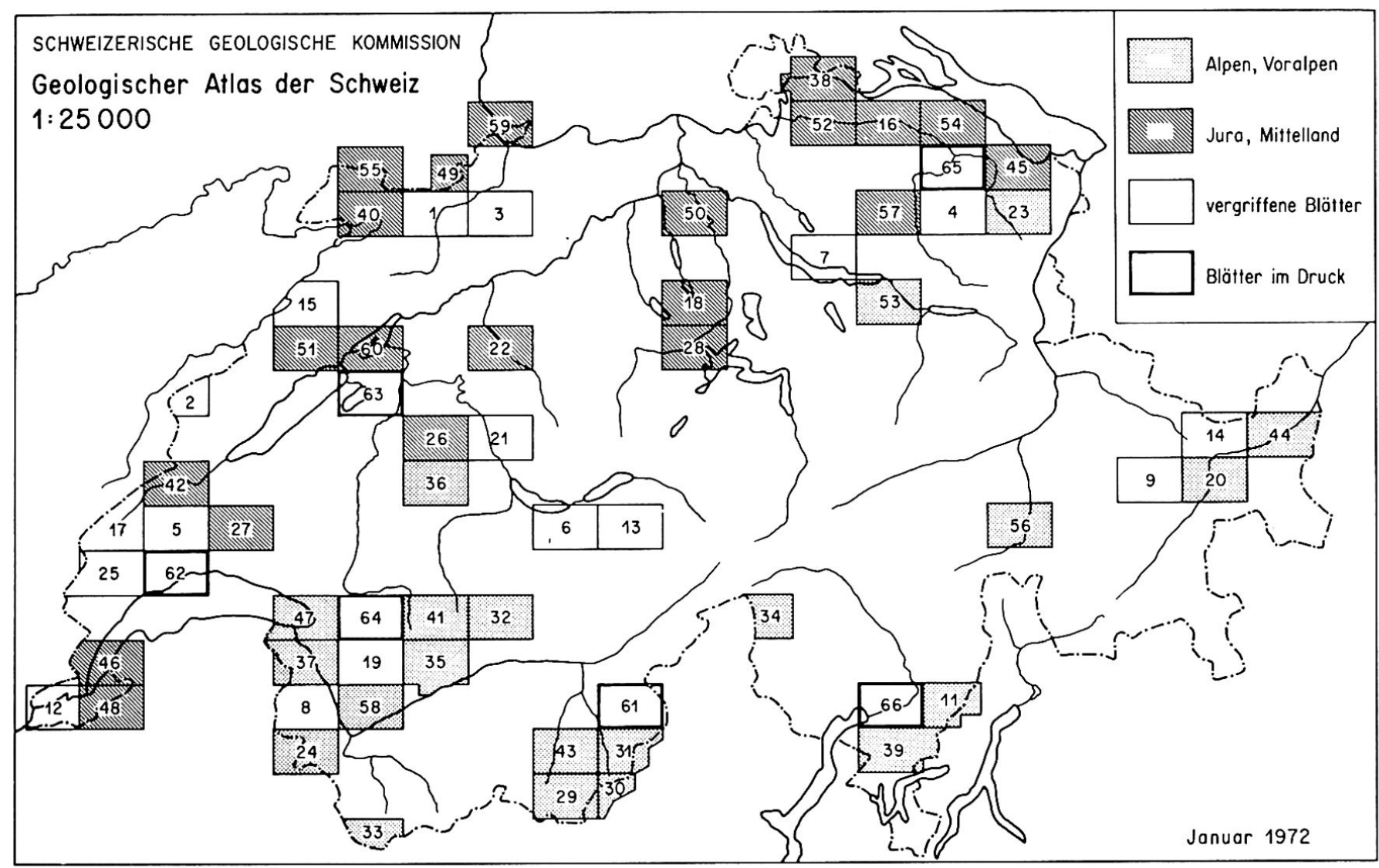


Nr. Blatt

$1^{*}$ 92-95 Movelier-Soyhières-Cour-

2* 276-277 La Chaux-Les Verrières

3* 96-99 Laufen-Bretzwil-ErschwilMümliswil

4* 218-221 Flawil-Herisau-Brunnadern-Schwellbrunn

5* 300-303 Mont-la-Ville-La SarrazMontricher-Cossonay

6* 395

7* 226-229

Lauterbrunnen

Mönchaltorf-Hinwil-

Wädenswil-Rapperswil

$8^{*} 483$

$9 * 423$

St-Maurice

Scaletta

$10 * 485$

Saxon-Morcles

11516

Iorio

12* 449_450 bis Dardagny_Vernier-Chancy-

$$
13 * 396
$$

$14 * 420$

Bernex

15* 114-117 Biaufond-Les Bois-

La Ferrière-St-Imier

16 56-59 Pfyn-Märstetten-Frauenfeld-Bussnang

$17 * 288,297$ bis Vallée de Joux (La Muratte-299 Les Mines-Le Lieu-Le Brassus-Le Sentier)

18 186-189 Beromünster-HochdorfSempach-Eschenbach

19* 477bis Diablerets (Chamossaire-

$\begin{array}{llll} & -480 & \text { Pillon-Gryon-Anzeindaz) } \\ 20 & 424 & \text { Zernez }\end{array}$

21 336-339 Münsingen-KonolfingenGerzensee-Heimberg

22 142-145 Fraubrunnen-WynigenHindelbank-Burgdorf

23 222-225 St. Gallen-Appenzell (Teufen-Trogen-AppenzellKobelwald)

24

$25 * 430-433$ Les Plats-Marchairuz-La Cure-Arzier-Gimel

26 332-335 Neuenegg-Oberbalm-

Schwarzenburg-Rüeggisberg 1953

27 304-307 Jorat (Echallens-Sottens-

Cheseaux-Corcelles-le-Jorat) 1952

28 202-205 Luzern (Rothenburg-

Emmen-Malters-Luzern)

29

30

31

32
$\mathrm{Jahr}$

$\begin{array}{ll} & 33 \\ 1930 & 34 \\ 1930 & 35 \\ & 36 \\ 1936 & \end{array}$

$1930 \quad 37$

$\begin{array}{lll}1930 & 37 & \\ & 38 & 1032 \\ 1935 & 39 & 1333\end{array}$

$1933 \quad 40 \quad 1085$

$$
41
$$

1934

1934

1935

1937

1939

1938

1938

1940

1946

1943

1941

1945

1940

1948

1949

1950

1949

1951

1950

1955

1953

1954

1954

1956
Jahr

1958

1957

1959

1961

1960

1961

1962

1963

1962

1963

Gedruckt auf Syntosil

Randa

1964

Scuol/Schuls-Tarasp 1963

Rorschach 1964

Coppet 1964

Montreux 1965

Genève 1965

Rodersdorf $\quad 1965$

Wohlen 1966

Val de Ruz 1968

Andelfingen $\quad 1967$

Linthebene $\quad 1969$

Weinfelden 1968

Bonfol 1969

Andeer 1971

Hörnli 1970

Dt de Morcles 1971

Basel 1970

Bieler See 1971

Simplon

Morges

Murten

Les Mosses

Bischofszell
$* *$

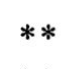

**

$* *$

$* *$
Bellinzona

$66 \quad 1313$

** Im Druck

Im weiteren sei noch darauf aufmerksam gemacht, daß im Sommer 1972 folgende zwei Karten erscheinen werden:

- Geologische Karte der Schweiz $1: 500000$

- Tektonische Karte der Schweiz 1:500 000

Beide Blätter sind auf der topographischen Unterlage der "Landeskarte der Schweiz 1:500 000" gedruckt. Der Verkaufspreis wird voraussichtlich Fr. 15.- pro Blatt betragen. 